\title{
Transferring Visual Prior for Online Object Tracking
}

\author{
Qing Wang, Feng Chen, Jimei Yang, Wenli Xu, and Ming-Hsuan Yang
}

\begin{abstract}
Visual prior from generic real-world images can be learned and transferred for representing objects in a scene. Motivated by this, we propose an algorithm that transfers visual prior learned offline for online object tracking. From a collection of realworld images, we learn an overcomplete dictionary to represent visual prior. The prior knowledge of objects is generic, and the training image set does not necessarily contain any observation of the target object. During the tracking process, the learned visual prior is transferred to construct an object representation by sparse coding and multiscale max pooling. With this representation, a linear classifier is learned online to distinguish the target from the background and to account for the target and background appearance variations over time. Tracking is then carried out within a Bayesian inference framework, in which the learned classifier is used to construct the observation model and a particle filter is used to estimate the tracking result sequentially. Experiments on a variety of challenging sequences with comparisons to several state-ofthe-art methods demonstrate that more robust object tracking can be achieved by transferring visual prior.
\end{abstract}

Index Terms-Object recognition, object tracking, sparse coding, transfer learning, visual prior.

\section{INTRODUCTION}

$\mathbf{O}$ BJECT tracking has been an important and active research topic in computer vision with numerous applications, including surveillance, traffic control, human-computer interfaces, and motion analysis, to name a few. The main challenge in developing a robust tracking algorithm is to account for large appearance variations of the target object and background over time. In this paper, we tackle this problem with both prior and online visual information. By learning visual prior from real-world images and transferring it to the tracking task, we

Manuscript received July 08, 2011; revised October 21, 2011 and February 14, 2012; accepted February 24, 2012. Date of publication April 05, 2012; date of current version June 13,2012. This work was supported in part by a Google Faculty Award, by NSF CAREER under Grant 1149783, and by NSF IIS under Grant 1152576. The work of Q. Wang and F. Chen was supported in part by the National Natural Science Foundation of China under Grant 61071131 and in part by the Beijing Natural Science Foundation under Grant 4122040. The work of J. Yang and M.-H. Yang was supported in part by the National Science Foundation (NSF) CAREER under Grant 1149783 and by the NSF Division of Information and Intelligent Systems (IIS) under Grant 1152576. The work of W. Xu was supported in part by the the National Key Basic Research and Development Program of China under Grant 2009CB320602. The associate editor coordinating the review of this manuscript and approving it for publication was Prof. Kenneth K. M. Lam.

Q. Wang, F. Chen, and W. Xu are with the National Laboratory for Information Science and Technology, Department of Automation, Tsinghua University, Beijing 100084, China (e-mail: qing-wang07@mails.tsinghua.edu.cn; chenfeng@mail.tsinghua.edu.cn; xuwl@mail.tsinghua.edu.cn).

J. Yang and M.-H. Yang are with the Department of Electrical Engineering and Computer Science, University of California at Merced, Merced, CA 95344 USA (e-mail: jyang44@ucmerced.edu; mhyang@ucmerced.edu).

Color versions of one or more of the figures in this paper are available online at http://ieeexplore.ieee.org.

Digital Object Identifier 10.1109/TIP.2012.2190085

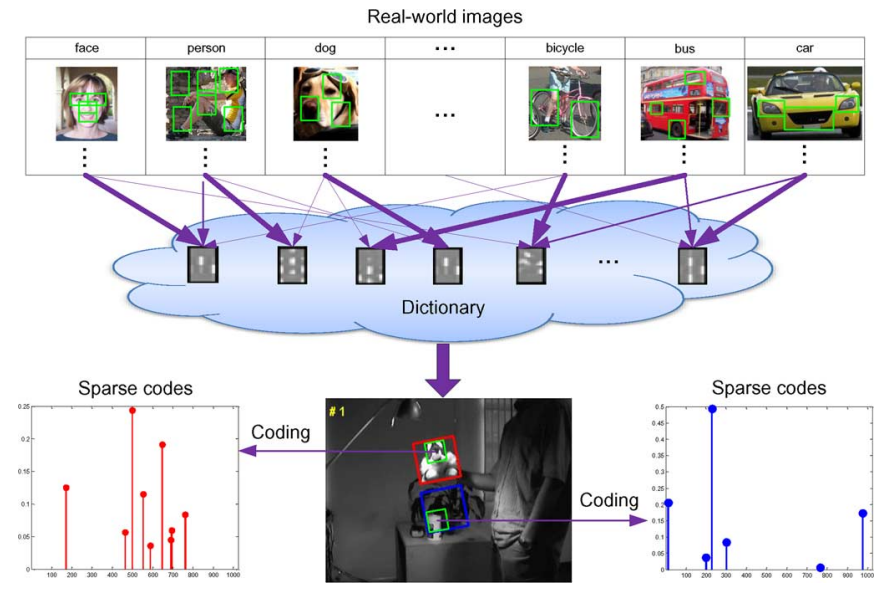

Fig. 1. Transferring visual prior to object tracking. We learn visual prior from real-world images and represent it with an overcomplete dictionary based on the SIFT features. The width of each arrow to the dictionary illustrates the contribution of one object class to one basis in the dictionary. With $\ell_{1} / \ell_{2}$ sparse coding on the learned dictionary, visual prior is transferred from the real-world images to the target object for tracking. The spare codes measure the contribution of the bases to represent an object patch and reflect the resemblance of the target and real-world object classes.

propose an adaptive tracking algorithm to account for appearance variations of the target and background.

The central theme of our approach is to exploit generic visual prior for object tracking. Although object tracking is usually an online task and visual information of the target may be scarce before the task starts, some useful prior can be still exploited offline particularly on the patch level. We note that there is a huge amount of real-world image data at our disposal. These images are likely to include similar holistic observations of the target object in the tracking task, but most likely they may not. Nevertheless, local patches from these images often share great similarity. Motivated by this, we learn generic visual prior from a large set of image data with an over-complete dictionary and sparse coding.

For visual tracking, we transfer the learned visual prior for object representation by sparse coding. Fig. 1 shows the prior representation and transfer processes in the proposed tracking algorithm. By filtering the sparse representation results at different scales, the corresponding object-level representation is obtained. With some samples from the target and background in the first frame, a classifier is initialized to distinguish them and the tracking task is formulated within the Bayesian inference framework. To account for the appearance changes of the target object and background during tracking, we update the classifier when new tracking results are obtained. Furthermore, to alleviate the visual drift problem during classifier update, we retain the initial classifier as a detector. In each frame, the observation model for Bayesian inference is constructed by a combination of the detector and the online classifier. 
For most tracking algorithms in the literature, either strong prior information of the target object is assumed or no prior knowledge is exploited. When an algorithm heavily depends on the prior, all views of the target should be known or a detailed geometric model is assumed before tracking, and the application domains of such algorithms are limited. In contrast, online tracking methods can be applied to numerous problems as no prior is required. However, the tracking results after a long duration period are usually unpredictable as only online visual information is used. Our algorithm exploits the strength of both approaches. In particular, we propose an algorithm that learns generic visual prior offline and transfers such knowledge to online object tracking.

The contributions of our tracking algorithm are summarized as follows. First, we learn a generic visual prior offline without assuming any specific knowledge of the target object for tracking. On the patch level, small images often share structural similarity, which motivates us to exploit such prior information offline and use it for online visual tracking. Second, the prior is represented by an overcomplete dictionary and learned by sparse coding from local patches. It is different from the widely used orthogonal dictionary (subspace) learned from holistic images by principal component analysis (PCA) or its variants. The nonorthogonal overcomplete dictionary learned from local patches makes our visual prior more effective for object description. Third, with the learned dictionary, $\ell_{1} / \ell_{2}$ sparse coding, and multiscale max pooling, a high-level object representation is constructed, and a simple classifier is capable of separating the target from the background.

\section{RELATED WORK}

There is a rich literature in object tracking, and a thorough review on this topic can be found in [28]. To deal with the problem of large object and background appearance variations, most recent tracking algorithms have focused on developing robust object representation schemes.

Since it is difficult to find a set of features that are invariant to appearance variations of target objects and backgrounds, learning algorithms have been adopted for this task. Based on a specific prior of the target, an object model can be learned offline. Black and Jepson [4] learn a subspace model to represent target objects at fixed views. In [5], Black et al. extend their subspace representation method to a mixture model that can better account for object appearance. Avidan [1] uses a set of vehicle and nonvehicle images collected offline to learn a classifier for car tracking. All these methods heavily depend on the specific prior. That is, these methods are developed for specific objects of interest. When all possible views of the target are known before tracking, object appearance models can be well constructed. However, in most real-world tracking applications, it is difficult to enumerate all possible appearance variations of objects. Therefore, such tracking algorithms have limited application domains.

Numerous adaptive appearance models have been recently proposed for object tracking. In these algorithms, object representation can be initialized and updated with online observations without any prior. Jepson et al. [13] learn a Gaussian mixture model via an online expectation maximization algorithm to account for target appearance variations during tracking. Aside from mixture models, incremental subspace methods based on PCA or its variants have been used for online object representation [17], [24]. To overcome the problem of partial occlusion, sparse representation has been also utilized for object tracking [21]. In [15], the authors extend the conventional particle filtering framework with multiple dynamic and observation models to account for target appearance variation caused by change of pose, illumination, scale, and partial occlusion. Object tracking has been also posited as a binary classification problem. Collins et al. [7] propose a method to select discriminative color features online for tracking, whereas Avidan [2] uses an online boosting method to classify pixels belonging to foreground and background. Recently, numerous approaches have been proposed to deal with the drift problem when updating the learned appearance model or classifier online with newly obtained tracking results. Grabner et al. [11] regard all the object information corresponding to the tracking results as unlabeled data and adapt a classifier within the semi-supervised learning framework. Babenko et al. [3] use multiple instance learning (MIL) to handle ambiguously labeled positive and negative data obtained online to reduce visual drift. Kalal et al. [14] propose a method to handle unbalanced samples that exploits the underlying structure to select positive and negative samples for online update. All these tracking algorithms do not assume any prior regarding the target object class and can be applied to numerous problems. However, persistent object tracking with these methods is difficult as it is not clear whether the updated visual information is correct or not (e.g., new observations may contain image regions from the background, and thus, incorrect information is updated).

Sparse coding algorithms model an observed example as a linear combination of a few elements from an overcomplete dictionary. The recent development of sparse coding/representation has attracted much interest and has been used in image denoising [8], [20], image classification [23], [27], and object tracking [21]. These methods have proven that a learning dictionary from data outperforms prechosen (fixed) ones (e.g., wavelet) since the former can significantly reduce reconstruction error [8]. Different from the representations based on PCA and its variants [17], [24], such sparse models do not impose that the bases in the dictionary be orthogonal, which allows more flexibility to adapt the representation to the data [19]. In [21], the sparse representation of a target object is achieved by optimizing an objective function, which includes two terms, i.e., one measures the reconstruction error and the other measures the sparsity. However, these methods are generative at its core (based on reconstruction error) for determining tracking results and are not equipped to distinguish target and background patches. In [23], the authors carry out sparse coding on raw image patches for image classification. In [27], the authors perform sparse coding on scale-invariant feature transform (SIFT) features [18] and achieve state-of-the-art performance for image classification on public benchmarks.

\section{LEARNING VisuAl PRIOR With SPARSE CODING}

We first present how generic visual prior can be learned from numerous images of diverse object classes. Although we can get a large number of real-world images, there is no straightforward method to exploit and represent generic visual prior in the 


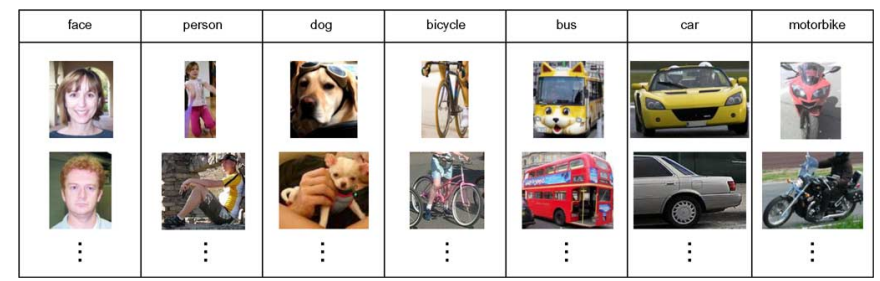

Fig. 2. Sample images for learning visual prior.

tracking literature. In this paper, we use sparse coding to learn the visual prior from large image sets of diverse objects with an overcomplete dictionary.

\section{A. Image Set}

This paper aims to bridge the gap between object recognition (based on visual prior) and object tracking (based on prior or online information). On the patch level, small images often share structural similarity. This is why we exploit such prior information offline from existing data sets and use it for online visual tracking. The VOC $2010^{1}$ and Caltech $101^{2}$ data sets, which consist of a large variety of objects, are used for learning visual prior. Without loss of generality, we use object classes that are common in surveillance scenarios from these two data sets, including nonrigid (e.g., face, person, and dog) and rigid (e.g., bicycle, bus, car, and motorbike) objects. Some images of these classes are shown in Fig. 2. It is worth noting that other related object images can be also used to learn a prior for specific tracking tasks.

\section{B. Learning Dictionary}

Since sparse coding based on SIFT descriptor has been proved to outperform sparse coding on raw image patches in computer vision [27], we also choose SIFT as the basic appearance descriptor in our tracking method. We extract the SIFT descriptors from overlapped patches of each grayscale image and learn the dictionary in an unsupervised manner. Let $X=\left[\mathbf{x}_{1}, \ldots, \mathbf{x}_{n}\right] \in \mathbb{R}^{m \times n}$ be the SIFT descriptors we extract from the image set, where $m$ and $n$ are the dimensionality of each SIFT descriptor and the number of SIFT descriptors, respectively. Denote $D=\left[\mathbf{d}_{1}, \ldots, \mathbf{d}_{k}\right] \in \mathbb{R}^{m \times k}(k \gg m)$ as the dictionary we want to learn; this problem can be formulated as

$$
\begin{aligned}
& \min _{D,\left\{\mathbf{a}_{i}\right\}} \frac{1}{2} \sum_{i=1}^{n}\left\|\mathbf{x}_{i}-D \mathbf{a}_{i}\right\|^{2}+\beta \sum_{i}^{n}\left\|\mathbf{a}_{i}\right\|_{1} \\
& \text { subject to }\left\|\mathbf{d}_{j}\right\|_{2}^{2} \leq 1 \quad \forall j \in\{1, \ldots, k\}
\end{aligned}
$$

where $\mathbf{a}_{i} \in \mathbb{R}^{k}$ is the sparse coefficient vector of $\mathbf{x}_{i}$ when encoded by the dictionary $D$. Parameter $\beta$ is a tradeoff between reconstruction error and sparsity. To enlarge the sparsity of the learned coefficient vector, we can increase $\beta$ and vice versa. The $\ell_{2}$-norm constraint on $\mathbf{d}_{j}$ is to prevent arbitrarily small values of $\mathbf{a}_{i}, \forall i \in\{1, \ldots, n\}$. Although there is a large number of SIFT descriptors extracted from the data set, $D$ is learned offline with the sparse coding method proposed in [16]. Some bases (column

${ }^{1} \mathrm{http}: / /$ pascallin.ecs.soton.ac.uk/challenges/VOC/voc2010/index.html

${ }^{2}$ http://www.vision.caltech.edu/Image_Datasets/Caltech101/ vectors) in $D$ are shown in the image form in Fig. 1. This dictionary $D$ contains generic structured information of different objects from numerous classes and is used to encode generic visual prior of objects.

Different from a prechosen or randomly selected codebook, the learned dictionary contains structural information of object appearance and can represent an object with less reconstruction error. Although it may be possible to represent an object with a prechosen or randomly selected codebook [10], [22], the coding coefficient vectors are likely to be denser and more sensitive to noise.

\section{TRANSFerring Visual PRIOR FOR OBJECT REPRESENTATION}

The learned visual prior is represented by the overcomplete dictionary $D$. We transfer this prior for object tracking by representing an object with $D$. For each SIFT descriptor inside an object region, a sparse coefficient vector is learned by performing $\ell_{1} / \ell_{2}$ sparse coding on the dictionary $D$. Then, an object is represented by applying multiscale max pooling on the coding results of all the local SIFT descriptors in their corresponding image region.

\section{A. $\ell_{1} / \ell_{2}$ Sparse Coding}

To represent an object, we first extract the SIFT descriptors from their image patches and then encode them with the learned dictionary. Let $X=\left[\mathbf{x}_{1}, \ldots, \mathbf{x}_{N}\right] \in \mathbb{R}^{m \times N}$ denote the SIFT descriptors extracted from an object image, the $\ell_{1} / \ell_{2}$ sparse coefficient vector $\mathbf{a}_{i} \in \mathbb{R}^{k}$ for coding $\mathbf{x}_{i}$ by the learned dictionary $D$ can be calculated by [29]

$$
\min _{\mathbf{a}_{i}} \frac{1}{2}\left\|\mathbf{x}_{i}-D \mathbf{a}_{i}\right\|_{2}^{2}+\lambda_{1}\left\|\mathbf{a}_{i}\right\|_{1}+\frac{\lambda_{2}}{2}\left\|\mathbf{a}_{i}\right\|_{2}^{2}
$$

where $\lambda_{1}$ and $\lambda_{2}$ are regularization parameters. When $\lambda_{2}=0$, it leads to the $\ell_{1}$-norm sparse coding problem, which has been widely used in [21] and [27]. The choice of $\lambda_{2}>0$ makes the problem in (2) become strictly convex. The coding results of all the descriptors in $X$ are denoted by a sparse coefficient matrix $A=\left[\mathbf{a}_{1}, \ldots, \mathbf{a}_{N}\right] \in \mathbb{R}^{k \times N}$, where each column of $\mathbf{A}$ denotes the coding result of the SIFT descriptor for an image patch.

With $\ell_{1} / \ell_{2}$ sparse coding, the SIFT descriptors from different objects can be encoded by different bases in the dictionary. The sparse coefficients from the dictionary reflect some structured information of the image, thereby facilitating inference of their class label (see Fig. 1). From this sparse coding process, the visual prior of generic objects is transferred to the tracking task.

\section{B. Comparison With Other Decomposition Methods}

The sparse coding method that we use for object representation is different from the PCA method and vector quantization (VQ) codebook. For object tracking, PCA has been widely used to learn specific prior (with orthogonal dictionary/subspace) [4], [5] and to model the target appearance online [24]. It is well known that in PCA, the training data are assumed to be Gaussian distributed and solved by $\ell_{2}$ optimization. When this assumption does not hold (e.g., due to occlusion), object representation using the dictionary learned by PCA is not effective. On the other hand, VQ-based codebook is also widely used for dictionary learning and encoding. Since VQ focuses on minimizing 
the overall reconstruction of data points, it is not effective in representing a diverse collection of images as there is a tradeoff between $\ell_{2}$ error and codebook size. Sparse coding can be regarded as a generalized VQ in which $\ell_{1}$ regularization allows $\mathbf{a}_{i}$ in (2) to have more than one nonzero element. Thus, sparse coding can achieve a much lower reconstruction error due to this less restrictive constraint. Compared with the $\ell_{1}$ sparse coding formulation, the use of $\ell_{1} / \ell_{2}$ constraints can lead to more stable coding results [29].

\section{Multiscale Max Pooling}

For the tracking task, we need to define an object-level feature for a target or a background sample over the sparse representation matrix A. There exist numerous methods for representing an object with a set of descriptors, and here we use a pooling function that operates on each row of $\mathbf{A}$ and obtain a vector $\mathbf{b} \in \mathbb{R}^{k}$. Since each row of $\mathbf{A}$ corresponds to the response of all local SIFT descriptors in $X$ to one specific basis in dictionary $D$, different pooling functions may generate different image statistics. To make the representation more robust to local spatial translations, we use the max pooling function on the absolute sparse codes

$$
b_{i}=\max \left\{\left|\mathbf{a}_{i, 1}\right|, \ldots,\left|\mathbf{a}_{i, N}\right|\right\}
$$

where $b_{i}$ is the $i$ th element of $\mathbf{b}$ and $\mathbf{a}_{i, j}$ is the element of the $i$ th row and the $j$ th column of $\mathbf{A}$. As discussed in [26] and [27], the max pooling process is well established with biophysical evidence in visual cortex and has been shown to be effective for object representation with local responses.

To preserve the spatial information and local invariance, we use multiscale max pooling to obtain the object-level representation. This pooling process searches across different locations and over different scales of the object image and combines all local maximum responses. In this paper, it is implemented by dividing the whole object image into $M$ nonoverlapped spatial cells, applying max pooling on the coding results of descriptors in each cell and concatenating the pooled features from all the spatial cells

$$
\mathbf{z}=\left[\mathbf{b}_{1}^{\top}, \ldots, \mathbf{b}_{M}^{\top}\right]^{\top}
$$

where $\mathbf{b}_{i}$ is the max pooling result of the $i$ th spatial cell, $M$ is the number of spatial cells, and $\mathbf{z} \in \mathbb{R}^{M k}$. With this process, we obtain a pyramid representation of an object, which is robust to local transformation.

\section{LEARNING ClASSIFIER}

After extraction of SIFT features, $\ell_{1} / \ell_{2}$ sparse coding, and multiscale max pooling, we obtain a spatial pyramid representation for each object image. With the overcomplete dictionary $D$, SIFT descriptors either from a target or a background object image can be well represented by its sparse coefficient vector with low reconstruction error. Therefore, different from [21] and [24], it is less effective to use observation models based on the reconstruction error for object tracking. With sparse coding and multiscale max pooling, images of different object classes are often represented by different bases of the learned dictionary. Therefore, it is easier to separate the samples from different classes. In this paper, we pose tracking as a binary classification problem, in which a linear classifier is learned to separate the target from the background. We use logistic regression to learn the classifier and use the classification score as our similarity measure for object matching. Although patches from different objects in the same category may be represented by similar sparse coefficients, the object-level representation based on multiscale max pooling exploits unique spatial characteristics of a target object. That is, our representation scheme consists of local feature their (based appearance) and responses geometric shape. Thus, the learned classifier is target specific, which can be used to discriminate a target from objects in the same or different categories. To account for appearance variations of the target and background during tracking, the classifier is updated with the most recent observations.

\section{A. Classifier Initialization}

To initialize the classifier, we need to collect some target (positive) and background (negative) samples with the sparse coding and multiscale max pooling process. In the first frame, when the target is labeled manually or by an object detector, we can get a set of target images with small location perturbations. To collect background samples, we first randomly draw samples from an annular region defined by $\gamma<\left\|\mathbf{1}-\mathbf{1}_{t}\right\|<\eta$ (i.e., $\gamma$ and $\eta$ are inner and outer radii), in which $\mathrm{l}_{t} \in \mathbb{R}^{2}$ is the location of the target sample and $l \in \mathbb{R}^{2}$ is the sample location. After extracting SIFT descriptors, representing these target and background images by sparse coding and multiscale max pooling, we obtain a set of training data denoted by $(\mathbf{z}, y) \in \mathbb{R}^{M k} \times\{+1,-1\}$, where $y$ is the class label. The linear classifier can be obtained by minimizing the cost function

$$
J(\mathbf{w})=\frac{1}{n_{s}} \sum_{i=1}^{n_{s}} \ell\left(y_{i}, \mathbf{w}, \mathbf{z}_{i}\right)+\frac{\lambda}{2}\|\mathbf{w}\|_{2}^{2}
$$

where $\mathbf{w}$ is the classifier parameter set we want to learn, $\ell$ is a loss function, and $n_{s}$ is the number of training samples. Parameter $\lambda>0$ controls the strength of the regularization term. In our method, we use the logistic loss function

$$
\ell(y, \mathbf{w}, \mathbf{z})=\log \left(1+e^{-y \mathbf{w}^{\top} \mathbf{z}}\right) .
$$

The corresponding classifier can be denoted by

$$
f(\mathbf{z})=\frac{1}{1+e^{-\mathbf{w}^{\top} \mathbf{z}}} .
$$

Once the classifier is initialized, the classification score can be utilized as the similarity measure for tracking.

\section{B. Online Update of the Classifier}

The image appearance of the target and background may change due to many factors such as pose, scale, illumination, and occlusion. Intuitively, object appearance remains the same only for a certain period of time, and eventually, the initialized model will be no longer accurate as time progresses. To account for image variations for robust object tracking, we update the classifier adaptively with new observations. With the recently obtained target observations and some negative samples extracted in the current frame, the classifier can be updated. We have experimented with incremental learning methods (e.g., 
stochastic gradient algorithm [6]) for online classifier update. However, such update methods are sensitive to the learning rate empirically, and thus, in our method, we update the classifier by retraining with the method presented in Section V-A. Since the number of samples for retraining is small, the classifier can be efficiently updated. As only the most recently obtained target observations are needed to be stored in the memory and the negative samples are collected in the current image frame, our algorithm does not have large memory requirement and is flexible to deal with long sequences.

\section{Proposed Tracking Algorithm}

In this paper, object tracking is carried out within the Bayesian inference framework. Given the observation set of the target $\mathbf{z}_{1: t}=\left\{\mathbf{z}_{1}, \ldots \mathbf{z}_{t}\right\}$ up to time $t$, the target state (motion parameter set) $\mathbf{x}_{t}$ can be determined by the maximum a posteriori estimation

$$
\hat{\mathbf{x}}_{t}=\arg \max _{\mathbf{x}_{t}} p\left(\mathbf{x}_{t} \mid \mathbf{z}_{1: t}\right)
$$

where $p\left(\mathbf{x}_{t} \mid \mathbf{z}_{1: t}\right)$ can be inferred by the Bayesian theorem in a recursive manner (with Markov assumption)

$$
p\left(\mathbf{x}_{t} \mid \mathbf{z}_{1: t}\right) \propto p\left(\mathbf{z}_{t} \mid \mathbf{x}_{t}\right) p\left(\mathbf{x}_{t} \mid \mathbf{z}_{1: t-1}\right)
$$

where $p\left(\mathbf{x}_{t} \mid \mathbf{z}_{1: t-1}\right)=\int p\left(\mathbf{x}_{t} \mid \mathbf{x}_{t-1}\right) p\left(\mathbf{x}_{t-1} \mid \mathbf{z}_{1: t-1}\right) d \mathbf{x}_{t-1}$. The tracking process is governed by a dynamic model, i.e., $p\left(\mathbf{x}_{t} \mid \mathbf{x}_{t-1}\right)$, and an observation model, i.e., $p\left(\mathbf{z}_{t} \mid \mathbf{x}_{t}\right)$.

A particle filter method [12] is adopted here to estimate the target state. In the particle filter, $p\left(\mathbf{x}_{t} \mid \mathbf{z}_{1: t}\right)$ is approximated by a finite set of samples $\left\{\mathbf{x}_{t}^{i}, i=1, \ldots, N_{s}\right\}$ with importance weights $\left\{\omega_{t}^{i}, i=1, \ldots N_{s}\right\}$. The candidate sample $\mathbf{x}_{t}^{i}$ is drawn from an importance distribution $q\left(\mathbf{x}_{t} \mid \mathbf{x}_{1: t-1}, \mathbf{z}_{1: t}\right)$, and the weight of the $i$ th sample is

$$
\omega_{t}^{i}=\omega_{t-1}^{i} \frac{p\left(\mathbf{z}_{t} \mid \mathbf{x}_{t}^{i}\right) p\left(\mathbf{x}_{t}^{i} \mid \mathbf{x}_{t-1}^{i}\right)}{q\left(\mathbf{x}_{t}^{i} \mid \mathbf{x}_{1: t-1}^{i}, \mathbf{z}_{1: t}\right)}
$$

where $q\left(\mathbf{x}_{t} \mid \mathbf{x}_{1: t-1}, \mathbf{z}_{1: t}\right)=p\left(\mathbf{x}_{t} \mid \mathbf{x}_{t-1}\right)$ in this paper.

The dynamic model $p\left(\mathbf{x}_{t} \mid \mathbf{x}_{t-1}\right)$ delineates the temporal correlation of the target states in consecutive frames. In our algorithm, we approximate the motion of a target between two consecutive frames with affine transformation. Let $\mathbf{x}_{t}$ be the 6-D vector where each parameter is independently modeled by a scalar Gaussian distribution centered at its counterpart of $\mathbf{x}_{t-1}$. Thus, the dynamic model is formulated as $p\left(\mathbf{x}_{t} \mid \mathbf{x}_{t-1}\right)=\mathcal{N}\left(\mathbf{x}_{t} ; \mathbf{x}_{t-1}, \Sigma\right)$, where $\Sigma$ is a diagonal covariance matrix whose elements are the variances of the affine parameters.

The observation model $p\left(\mathbf{z}_{t} \mid \mathbf{x}_{t}\right)$ denotes the likelihood of $\mathbf{x}_{t}$ generating observation $\mathbf{z}_{t}$. It plays a key role for robust tracking because it directly corresponds to the core challenge of tracking, i.e., unpredictable variations such as appearance or background changes. When the classifier is available, the observation model can be constructed as

$$
p\left(\mathbf{z}_{t} \mid \mathbf{x}_{t}\right) \propto c_{t}
$$

where $c_{t}=f\left(\mathbf{z}_{t}\right)$ is the classification score at time $t$. To alleviate the visual drift problem during update, we retain the initial classifier trained in the first frame as a detector. In each frame, the total classification score can be calculated by

$$
c_{t}^{*}=(1-r) c_{1}+r c_{t}
$$

where $c_{1}$ and $c_{t}$ are the classification scores of the detector initialized in the first frame and the adaptive classifier at time $t$, respectively. We denote $c_{t}^{*}$ as the final classification score at time $t$, and $r$ is a predefined constant, which determines the confidence of our adaptive classifier. That is, the adaptive classifier helps account for rapid appearance change while the detector alleviates the drift problem. With the prior learned offline, the online tracking algorithm is carried out, as summarized in Algorithm 1.

Algorithm 1 Summary of the Online Tracking Algorithm.

1: Input: Video frames $F_{1}, \ldots, F_{T}$.

2: Output: Target states $\mathbf{x}_{1}, \ldots, \mathbf{x}_{T}$.

3: for $t=1, \ldots, T$ do

4: if $t=1$ then

5: Transfer prior for object representation.

6: $\quad$ Initialize the classifier with parameter set $\mathbf{w}_{1}$.

7: else

8: $\quad$ Transfer prior for object representation.

9: $\quad$ Estimate $\mathbf{x}_{t}$ using particle filtering.

10: Store target observation corresponding to $\mathbf{z}_{t}$.

11: if The number of target observations is equal to some predefined threshold then

12: Collect a number of negative samples in the current frame.

13: Use the target observations (positive samples) and negative samples to update $\mathbf{w}_{t}$.

14: Clear the target observation set.

15: else

16: $\quad \mathbf{w}_{t}=\mathbf{w}_{t-1}$

17: end if

18: end if

19: end for

\section{EXPERIMENTS}

We evaluate our tracker on 12 challenging image sequences (some of them are publicly available) against several state-ofthe-art algorithms. The challenging factors in these sequences include pose change, illumination change, occlusion, cluttered background, image blur, and camera motion. 


\section{A. Implementation}

The proposed algorithm consists of an offline prior learning module and an online object tracking component. In the offline phase, the SIFT descriptors are densely extracted from $16 \times 16$ patches on a grid with step size of 8 pixels from each selected image (based on intensity). With about 200000 SIFT descriptors, we learn a $128 \times 1024$ dictionary. For convenience of further analysis, we add a label to each basis vector in the dictionary to show which object class contributes most to this basis. (Note that a basis vector may be learned by the SIFT descriptors from different object classes, which is illustrated in Fig. 1.)

For multiscale max pooling in the online tracking phase, we use three spatial levels and set the numbers of cells on three levels to be 1, 4, and 9, respectively. Therefore, the dimensionality of each object representation is $\mathbf{z} \in \mathbb{R}^{14336}$. In the first frame, linear classifier $f$ is initialized with 60 target samples and 60 background samples. Classifier parameter $\mathbf{w}$ is updated every ten frames as a tradeoff of effectiveness and efficiency. Confidence ratio $r$ is set to be 0.8 , and the number of particles is 300 in all experiments. ${ }^{3}$

\section{B. Baseline Experiments}

In the Sylvester sequence [24], the target (plush toy) undergoes pose and illumination change. The results in Fig. 3(a) show that the proposed algorithm is able to track the target well. In the Wall-E sequence shown in Fig. 3(b), the proposed algorithm is able to account for appearance variation due to drastic scale and pose change (3-D motion) of the target. Note that we do not have the same object images in the training set. These two experiments demonstrate that the learned generic visual prior can be used to represent different kinds of objects.

To illustrate how the visual prior is transferred for object tracking, we plot the probability of learned bases of each object class being selected to represent the target. Since we can determine which object class contributes most to one basis when learning the dictionary offline, we mark each basis with the corresponding class label. Thus, during tracking, the class label of each basis being used to encode each local SIFT descriptor can be identified. With the coding coefficients, we can compute the probability of the visual prior transferred from each training object class to the corresponding local descriptor. For the Sylvester and Wall-E sequences, we select one image patch (marked by a blue polygon) from the target region and plot the sparse coding results on the dictionary and the histograms of prior being transferred from each training object class in Fig. 3(a) and (b). We note that the visual prior of the $d o g$ object class is used in the Sylvester sequence; the visual prior of the bus is exploited most in the Wall-E sequence. The results can be explained by the fact that some patches of these targets bear significant resemblance to the object classes in the training images (i.e., plush toy versus dog and robot car versus bus).

\section{Qualitative Evaluation}

We compare our tracker with several object tracking algorithms, i.e., the incremental visual tracker (IVT) [24], the variance ratio tracker (VRT) [7], the L1 tracker (L1T) [21], the MIL

${ }^{3}$ Videos and source codes are available at http://faculty.ucmerced.edu/ mhyang/papers/tip11a.html.
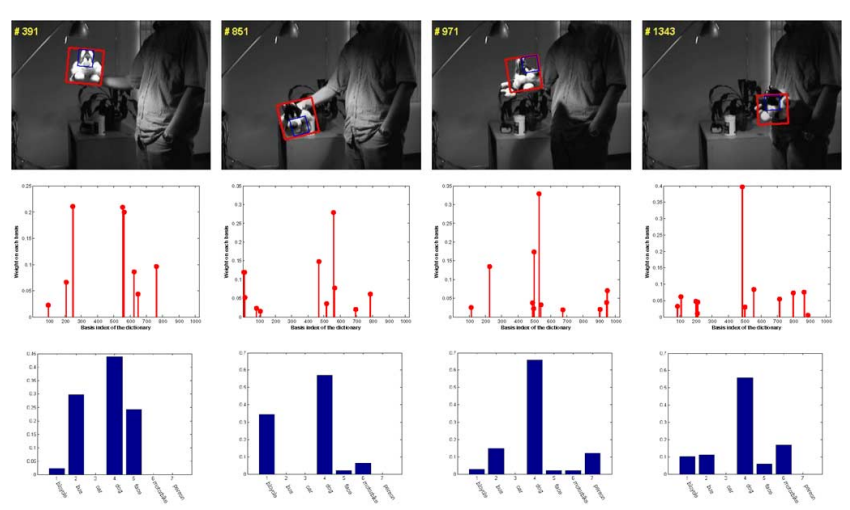

(a)
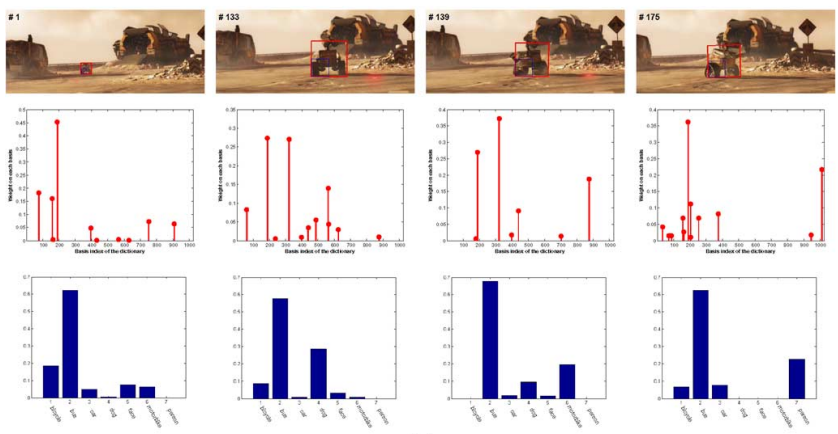

(b)

Fig. 3. Tracking results and prior transfer of the Sylvester and Wall-E sequences. The second rows of (a) and (b) show the coding results of an image patch inside the object region over time, which reflects the weight changes of each basis in the dictionary that an image patch is dependent on. The third rows of (a) and (b) illustrate the prior transfer from the real-world images to the target object (SIFT descriptor extracted from the image patch in the blue polygon). The numbers on the $x$-coordinate refer to different classes of training data. The $y$-coordinate illustrates how much prior is transferred to the tracking object from each object class.

tracker (MILT) [3], the visual tracking decomposition tracker (VTD) [15], and the tracking-learning-detection (TLD) method [14]. We obtain the tracking results of these compared methods with the codes provided by the authors. For the IVT, L1T, VTD, and our methods, we use the same parameters for particle filtering. Both the IVT and L1T methods learn dictionaries online from test videos. The bases learned in the IVT method are orthogonal to each other, and the number of bases is not larger than the dimensionality of the feature vector. On the contrary, the dictionary in the L1T method is overcomplete. The TLD method is equipped with a detection procedure to help find the object after occlusion. The IVT, L1T, and VTD trackers are generative methods, whereas the others are discriminative trackers. We also use experiments to show that the prior transfer is vital for robust object tracking. To this end, we implement a tracking algorithm (referred to as SIFTT) wherein the SIFT descriptors are directly used for object representation by multiscale max pooling. All the other modules are the same as our algorithm. In the following sections, we present some representative tracking results.

Pose Change: The Freeman sequence is used to test the performance of our tracker in handling pose change. There is also a significant scale change when the target walks toward the camera. From the tracking results illustrated in Fig. 4, we note that our method and the TLD method perform well whereas the 


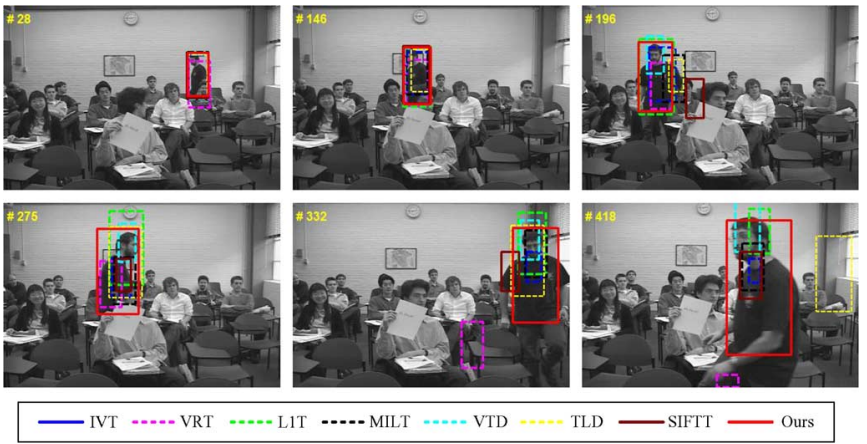

Fig. 4. Tracking results of the Freeman sequence.
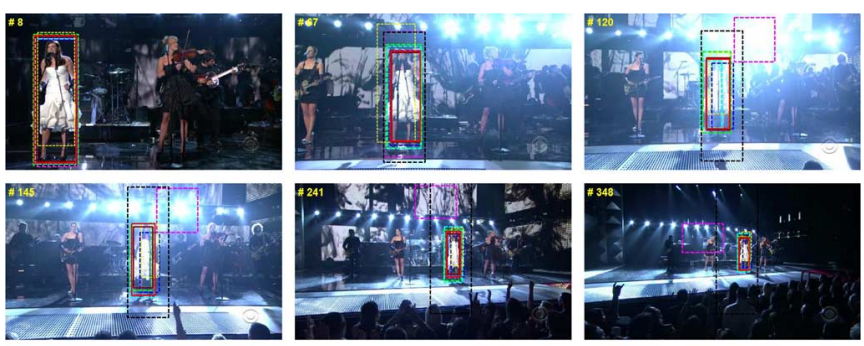

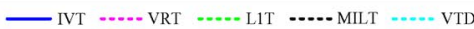
TLD $\longrightarrow$ SIFTT Ours

Fig. 5. Tracking results of the singer sequence.
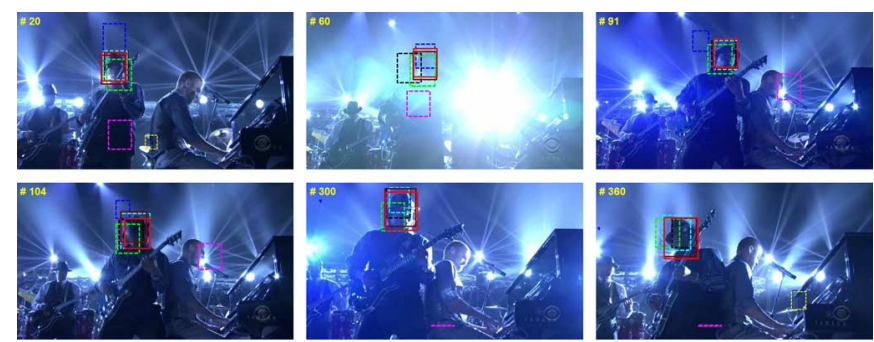

—IVT TLD — SIFTT — Ours

Fig. 6. Tracking results of the shaking sequence.

IVT, L1T, MILT, VTD, and SIFTT have some tracking errors. The VRT method gradually fails.

Illumination Change: We use the singer and shaking sequences [15] to evaluate whether our tracker is able to handle drastic illumination change. Some representative tracking results are shown in Figs. 5 and 6. In both sequences, our tracker performs well when compared with the VTD method. In the singer sequence, there are large-scale changes of the target and unknown camera motion in addition to illumination change. The VRT method fails to track the target when illumination changes. The MILT and TLD algorithms also succeed in tracking the target but do not deal with the scale changes well. The IVT, L1T, and SIFTT methods have similar tracking results as our tracker. In the shaking sequence, the target undergoes pose variation besides illumination change. The L1T, MILT, and SIFTT algorithms are also able to track the target, whereas the IVT, VRT, and TLD methods drift from the target quickly. Our tracker uses an online update mechanism to account for the appearance variation of the target and background over time and retains a detector to alleviate visual drift problem. In addition, the object representation based on sparse coding and
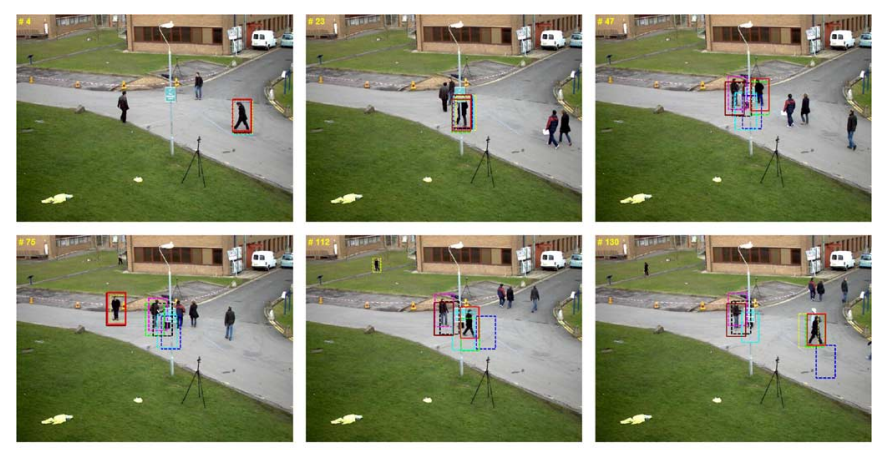

—IVT

VTD

TLD $\longrightarrow$ SIFTT

Fig. 7. Tracking results of the PETS2009 sequence.

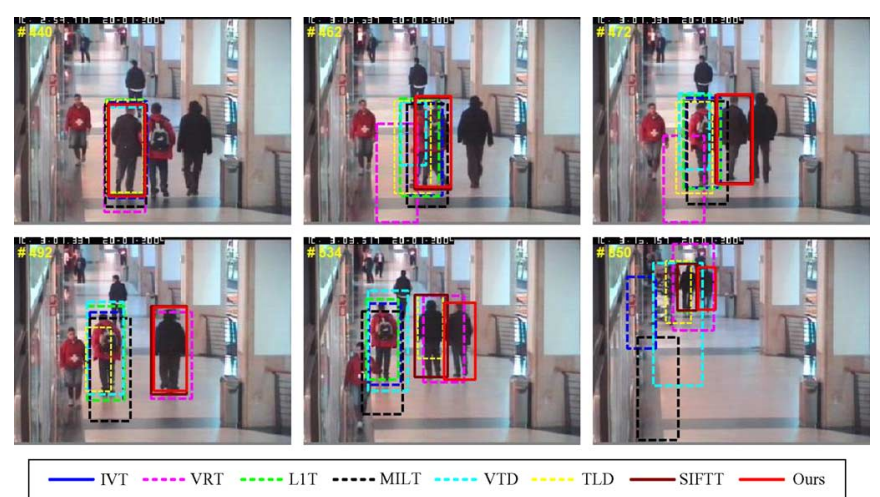

Fig. 8. Tracking results of the CAVIAR sequence.

multiscale max pooling is less sensitive to illumination and pose change, thereby achieving good tracking performance.

Occlusion: We use the PETS2009 and CAVIAR sequences to test the performance of our tracker when the target object undergoes heavy occlusion. In the PETS2009 sequence, there is also an out-of-plane pose change besides heavy occlusion. The tracking results presented in Fig. 7 show that our tracking method and the TLD tracker succeed in tracking the target object after heavy occlusion, whereas the others all fail. In this sequence, the geometric shapes and local responses of the target objects are different from the other objects. Consequently, they can be used to differentiate the other objects. For the TLD method, it has a detection procedure, thereby also succeeding in tracking after occlusion. In the CAVIAR sequence (see Fig. 8), it is difficult to keep track of the target after occlusion because there are other objects with similar appearances in the scene. The IVT, L1T, MILT, VTD, TLD, and SIFTT methods do not perform well, whereas the VRT algorithm performs slightly better. In contrast, our method exploits visual prior and represents objects by coding results of local image patches described by SIFT features for learning a target-specific classifier. Thus, our method is able to discriminate the target from others in the same object category and performs well in this sequence. The initial classifier also facilitates the proposed method to keep track of the target when heavy occlusion occurs.

Background Clutter: We use the box [25], board [25], and $N B A$ sequences to evaluate our tracker in handling background clutter. In the box sequence shown in Fig. 9, there are also partial occlusions, which adds difficulty for object tracking. From 

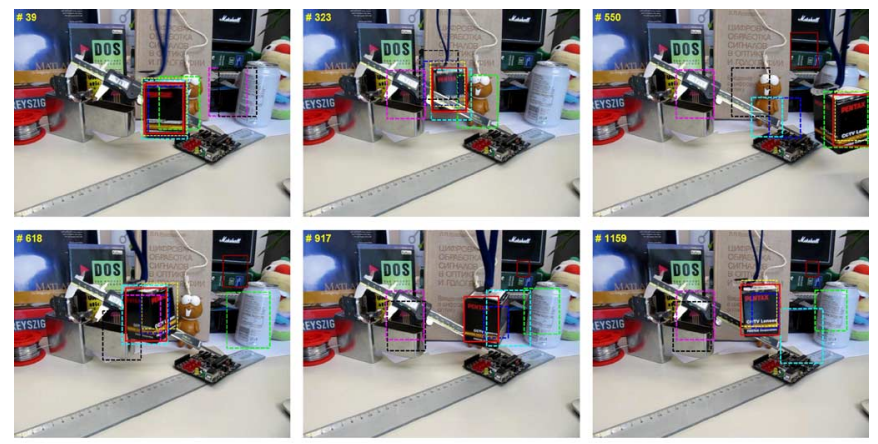

—IVT

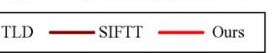

Fig. 9. Tracking results of the box sequence.
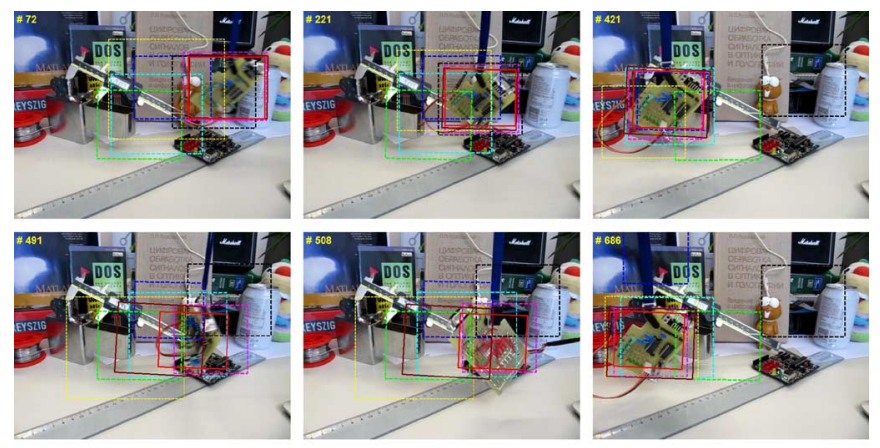

— IVT $\ldots . . .$. VRT $\ldots . .$. LIT $\ldots . .$. MIT

Fig. 10. Tracking results of the board sequence.
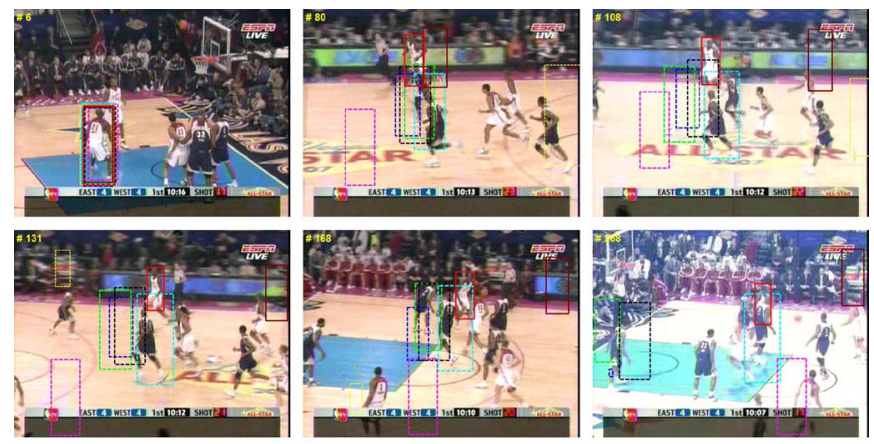

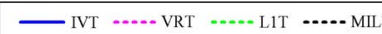

TLD - SIFTT - Ours

Fig. 11. Tracking results of the $N B A$ sequence.

the tracking results in Fig. 9, we observe that our method and the TLD tracker perform better than the other trackers. In the board sequence shown in Fig. 10, our tracker performs well when the target undergoes out-of-plane rotation (3-D motion). All the other methods lose track of the target in some frames. In the $N B A$ sequence (see Fig. 11), the target object is similar to other objects in the scene. The IVT, VRT, L1T, MILT, VTD, TLD, and SIFTT methods all lose track of the target gradually, whereas our tracker succeeds in most of the frames except toward the end when the target undergoes occlusion for numerous frames.

Image Blur and Low Contrast: Figs. 12 and 13 demonstrate the performance of these trackers in handling scenarios with image blur and low foreground-background contrast. In the car sequence shown in Fig. 12, the target also undergoes partial
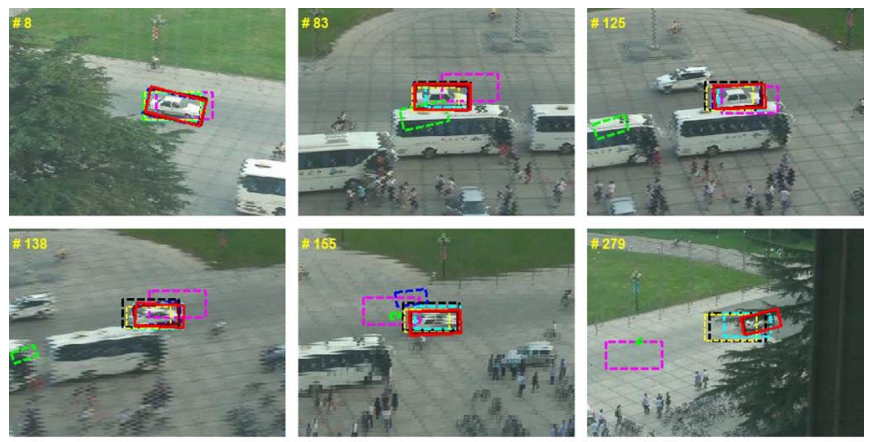

- IVT -.... VRT

Fig. 12. Tracking results of the car sequence.
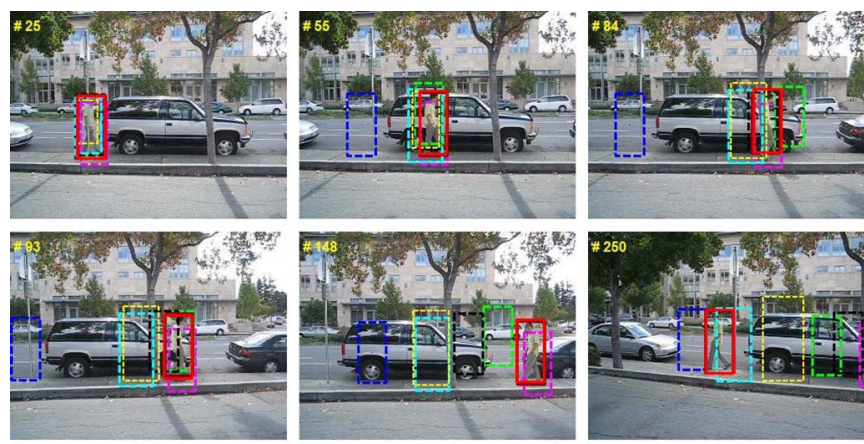

——IVT

TLD — SIFTT — Ours

Fig. 13. Tracking results of the David sequence.

occlusion and pose variation other than image blur caused by camera motion. Our tracker performs well in this sequence, whereas the L1T method quickly fails when the car is partially occluded by a bus. It can be explained by the fact that global intensity features used in the L1T method cannot discriminate the car object from buses that have similar holistic appearance. The IVT and VRT methods perform better than L1T, but they lose track of the target when image blur occurs. The MILT, VTD, and TLD methods are able to track the target in this sequence although with some errors in the last frames. The SIFTT method provides similar tracking result as ours. In the David sequence shown in Fig. 13, the contrast between the target and the background is low. In addition, there is severe occlusion and a large pose change of the target. The IVT method quickly fails after David is partially occluded by a pole, and the VTD and TLD methods gradually fail when David walks in front of the van. The MILT method fails after the target walks behind a tree. The VRT and L1T methods perform better but also gradually fail after the target turns around. On the other hand, our tracker and the SIFTT method succeed throughout this sequence.

\section{Quantitative Evaluation}

Aside from the qualitative comparison, we compute the tracking success rate and center location error using the ground truth manually labeled at every five frames. We employ the criterion used in the PASCAL Visual Object Classes challenge [9] to determine whether each tracking result is a success. Given the tracked bounding box $R O I_{T}$ and the ground truth bounding box $R O I_{G}$, the score is defined as score $=\operatorname{area}\left(R O I_{T} \bigcap R O I_{G}\right) / \operatorname{area}\left(R O I_{T} \bigcup R O I_{G}\right)$. The 

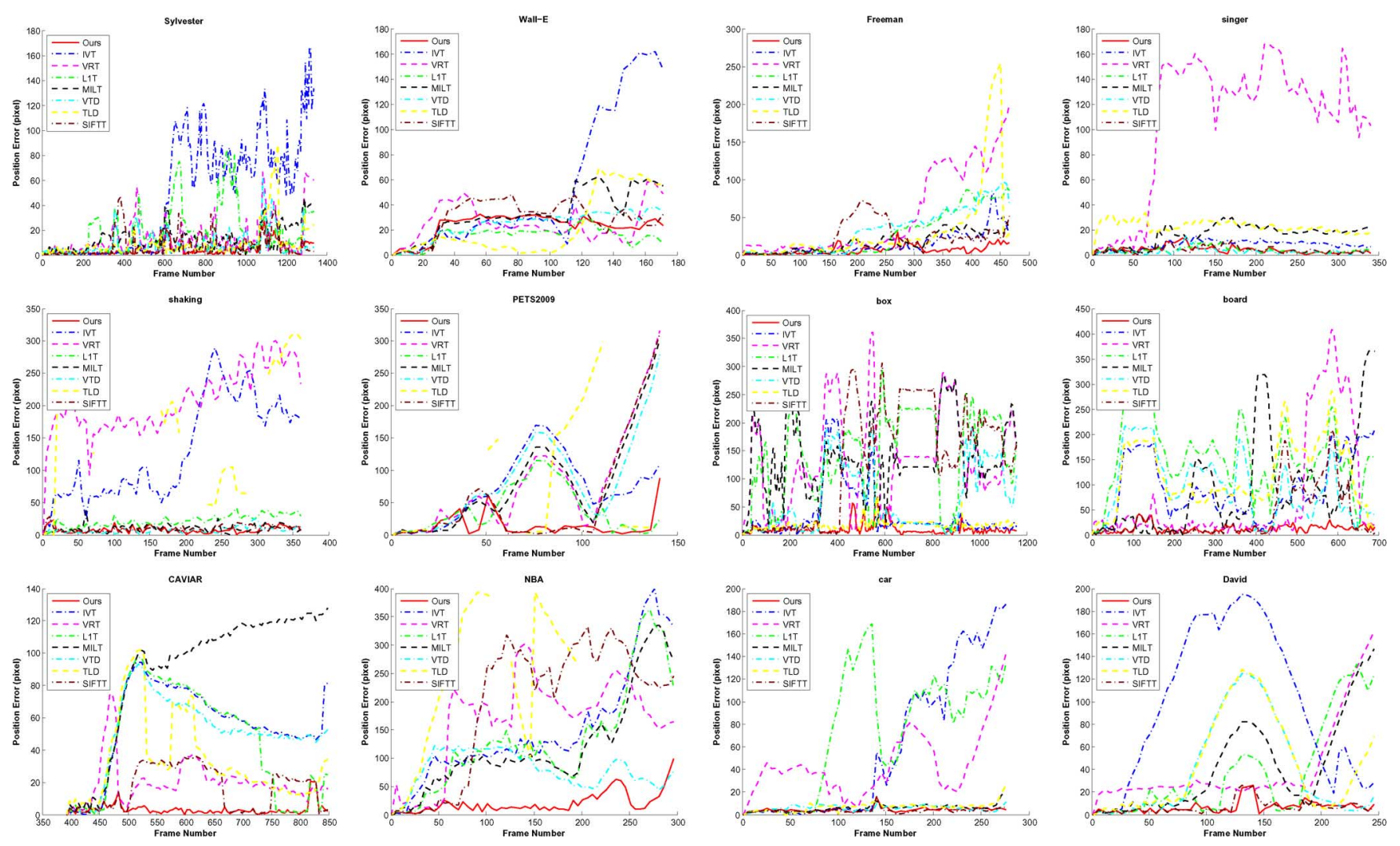

Fig. 14. Error plots of the test sequences.

TABLE I

Success Rates (\%). The Best Two Results Are Presented IN BOLD FACE AND ITALIC FONTS

\begin{tabular}{|c||c|c|c|c|c|c|c|c|}
\hline & IVT & VRT & L1T & MILT & VTD & TLD & SIFTT & Ours \\
\hline Sylvester & $\mathbf{4 5}$ & 72 & 36 & 68 & 79 & 86 & 81 & $\mathbf{9 1}$ \\
\hline Wall-E & 11 & 8 & 51 & 8 & 20 & 70 & 17 & $\mathbf{8 4}$ \\
\hline Freeman & 43 & 29 & 55 & 37 & 39 & 49 & 34 & $\mathbf{9 6}$ \\
\hline singer & 56 & 20 & $\mathbf{1 0 0}$ & 23 & 99 & 36 & 92 & $\mathbf{1 0 0}$ \\
\hline shaking & 3 & 0 & 27 & 88 & $\mathbf{9 6}$ & 5 & 93 & 93 \\
\hline PETS2009 & 21 & 21 & 24 & 24 & 14 & 41 & 34 & $\mathbf{5 9}$ \\
\hline CAVIAR & 15 & 17 & 30 & 15 & 13 & 14 & 47 & 96 \\
\hline box & 28 & 9 & 11 & 4 & 43 & 90 & 35 & $\mathbf{9 3}$ \\
\hline board & 21 & 77 & 9 & 44 & 32 & 13 & 75 & 99 \\
\hline NBA & 12 & 15 & 13 & 12 & 7 & 7 & 20 & $\mathbf{5 7}$ \\
\hline car & 50 & 9 & 29 & 95 & $\mathbf{9 6}$ & 95 & 95 & $\mathbf{9 6}$ \\
\hline David & 12 & 2 & 42 & 30 & 40 & 28 & 90 & $\mathbf{9 2}$ \\
\hline
\end{tabular}

tracking result in one frame is considered as a success when this score is above 0.5 . Table I shows the tracking results in terms of success rates. The center location error is defined as the distance between the central locations of the tracked target and the ground truth. The tracking results in terms of center location errors are illustrated in Fig. 14. The results demonstrate that our tracking algorithm performs well against the other state-of-the-art methods. Note that in the Sylvester, Wall-E, box, and board sequences, no samples of these object classes are included in the training set. The experimental results demonstrate that the learned visual prior is generic and can be applied to different tracking tasks. The comparisons also demonstrate the necessity of learning and transferring visual prior instead of using the SIFT descriptor directly for object representation.

\section{CONCLUSION}

This paper has exploited generic visual prior learned from real-world images for online tracking of specific objects. On the patch level, small images often share structural similarity, and such prior information can be learned offline and used for modeling objects in online visual tracking. We have presented an effective method that learns and transfers visual prior for robust object tracking. With a large set of natural images, we represent visual prior with an overcomplete dictionary. We transfer the learned prior to tracking tasks by sparse coding and represent the object with the multiscale max pooling method. With newly arrived samples of the target and background, a classifier is learned online to discriminate the target object from the background and a particle filter algorithm is utilized to estimate the target state sequentially. Compared with the related state-of-the-art tracking methods, the proposed tracking algorithm is demonstrated to robustly perform in complex environments where the target and background undergo different kinds of variations.

\section{ACKNOWLEDGMENT}

This work was performed in part while Q. Wang was a visiting student at the University of California at Merced.

\section{REFERENCES}

[1] S. Avidan, "Support vector tracking," in Proc. IEEE Conf. Comput. Vis. Pattern Recog., 2001, pp. 184-191.

[2] S. Avidan, "Ensemble tracking," in Proc. IEEE Conf. Comput. Vis. Pattern Recog., 2005, vol. 2, pp. 494-501.

[3] B. Babenko, M.-H. Yang, and S. Belongie, "Visual tracking with online multiple instance learning," in Proc. IEEE Conf. Comput. Vis. Pattern Recog., 2009, pp. 983-990.

[4] M. Black and A. Jepson, "Eigentracking: Robust matching and tracking of articulated objects using a view-based representation," in Proc. Eur. Conf. Comput. Vis., 1996, pp. 329-342. 
[5] M. J. Black, D. J. Fleet, and Y. Yacoob, "A framework for modeling appearance change in image sequences," in Proc. IEEE Int. Conf. Comput. Vis., 1998, pp. 660-667.

[6] A. Bordes, L. Bottou, and P. Gallinari, "SGD-QN: Careful quasi-Newton stochastic gradient descent," J. Mach. Learn. Res., vol. 10, pp. 1737-1754, 2009.

[7] R. T. Collins, Y. Liu, and M. Leordeanu, "Online selection of discriminative tracking features," IEEE Trans. Pattern Anal. Mach. Intell., vol. 27, no. 10, pp. 1631-1643, Oct. 2005.

[8] M. Elad and M. Aharon, "Image denoising via sparse and redundant representations over learned dictionaries," IEEE Trans. Image Process., vol. 15, no. 12, pp. 3736-3745, Dec. 2006.

[9] M. Everingham, L. Van Gool, C. Williams, J. Winn, and A. Zisserman, "The Pascal visual object classes (voc) challenge," Int. J. Comput. Vis., vol. 88, no. 2, pp. 303-338, Jun. 2010.

[10] P. Geurts, D. Ernst, and L. Wehenkel, "Extremely randomized trees," Mach. Learn., vol. 63, no. 1, pp. 3-42, 2006.

[11] H. Grabner, C. Leistner, and H. Bischof, "Semi-supervised on-line boosting for robust tracking," in Proc. Eur. Conf. Comput. Vis., 2008, pp. 234-247.

[12] M. Isard and A. Blake, "CONDENSATION-Conditional density propagation for visual tracking," Int. J. Comput. Vis., vol. 29, no. 1, pp. 5-28, 1998.

[13] A. D. Jepson, D. J. Fleet, and T. F. El-Maraghi, "Robust online appearance models for visual tracking," IEEE Trans. Pattern Anal. Mach. Intell., vol. 25, no. 10, pp. 1296-1311, Oct. 2003.

[14] Z. Kalal, J. Matas, and K. Mikolajczyk, "P-N learning: Bootstrapping binary classifiers by structural constraints," in Proc. IEEE Conf. Comput. Vis. Pattern Recog., 2010, pp. 49-56.

[15] J. Kwon and K. Lee, "Visual tracking decomposition," in Proc. IEEE Conf. Comput. Vis. Pattern Recog., 2010, pp. 1269-1276.

[16] H. Lee, A. Battle, R. Raina, and A. Ng, "Efficient sparse coding algorithms," Adv. Neural Inf. Process. Syst., vol. 19, pp. 801-808, 2007.

[17] X. Li and W. Hu, "Robust visual tracking based on incremental tensor subspace learning," in Proc. IEEE Int. Conf. Comput. Vis., 2007, pp. $1-8$.

[18] D. Lowe, "Distinctive image features from scale-invariant keypoints," Int. J. Comput. Vis., vol. 60, no. 2, pp. 91-110, Nov. 2004.

[19] J. Mairal, F. Bach, and J. Ponce, "Task-driven dictionary learning," INRIA, Rocquencourt, France, Tech. Rep. 7400, 2010.

[20] J. Mairal, F. Bach, J. Ponce, G. Sapiro, and A. Zisserman, "Nonlocal sparse models for image restoration," in Proc. IEEE Int. Conf. Comput. Vis., 2009, pp. 2272-2279.

[21] X. Mei and H. Ling, "Robust visual tracking using $\ell 1$ minimization," in Proc. IEEE Int. Conf. Comput. Vis., 2009, pp. 1436-1443.

[22] F. Moosmann, B. Triggs, and F. Jurie, "Fast discriminative visual codebooks using randomized clustering forests," Adv. Neural Inf. Process. Syst., pp. 985-992, 2006.

[23] R. Raina, A. Battle, H. Lee, B. Packer, and A. Ng, "Self-taught learning: Transfer learning from unlabeled data," in Proc. Int. Conf. Mach. Learn., 2007, pp. 759-766.

[24] D. Ross, J. Lim, R.-S. Lin, and M.-H. Yang, "Incremental learning for robust visual tracking," Int. J. Comput. Vis., vol. 77, no. 1-3, pp. $125-141,2008$

[25] J. Santner, C. Leistner, A. Saffari, T. Pock, and H. Bischof, "Prost: Parallel robust online simple tracking," in Proc. IEEE Conf. Comput. Vis. Pattern Recog., 2010, pp. 723-730.

[26] T. Serre, L. Wolf, and T. Poggio, "Object recognition with features inspired by visual cortex," in Proc. IEEE Conf. Comput. Vis. Pattern Recog., 2005, vol. 2, pp. 994-1000.

[27] J. Yang, K. Yu, Y. Gong, and T. Huang, "Linear spatial pyramid matching using sparse coding for image classification," in Proc. IEEE Conf. Comput. Vis. Pattern Recog., 2009, pp. 1794-1801.

[28] A. Yilmaz, O. Javed, and M. Shah, "Object tracking: A survey," $A C M$ Comput. Surveys, vol. 38, no. 4, p. 13, 2006.

[29] H. Zou and T. Hastie, "Regularization and variable selection via the elastic net," J. Roy. Statist. Soc., Ser. B, vol. 67, no. 2, pp. 301-320, 2005.

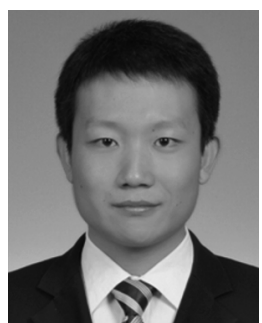

Qing Wang received the B.S. degree in automation from Northwestern Polytechnical University, Xi'an, China, in 2007, and is currently working toward the $\mathrm{Ph} . \mathrm{D}$. degree in automation from Tsinghua University, Beijing, China.

His current research interests include computer vision and machine learning.

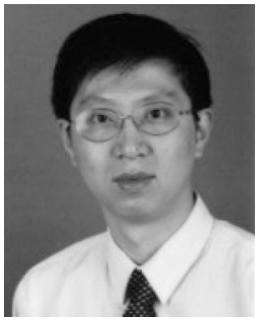

Feng Chen received the B.S. and M.S. degrees in automation from St. Petersburg State Polytechnical University, St. Petersburg, Russia, in 1994 and 1996, respectively, and the Ph.D. degree in automation from Tsinghua University, Beijing, China, in 2000.

$\mathrm{He}$ is a Professor at Tsinghua University. His research interests are probabilistic graphical models and computer vision.

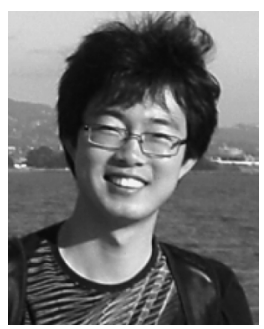

Jimei Yang received the B.S. degree in electronics and information science from the China Agricultural University, Beijing, China, in 2006, the M.S. degree in automation from the University of Science and Technology of China, Beijing, China, in 2009, and is currently working toward the Ph.D. degree in computer science from the University of California at Merced.

From 2007 to 2009 , he was with the Institute of Automation, Chinese Academy of Sciences, Beijing, China, as a Research Assistant.

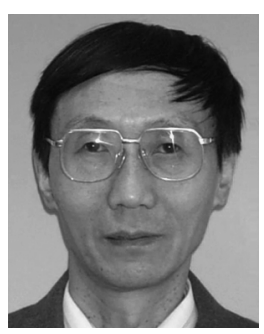

Wenli Xu received the B.S. degree in electrical engineering and the M.S. degree in automatic control engineering from Tsinghua University, Beijing, China, in 1970 and 1980, respectively, and the Ph.D. degree in electrical and computer engineering from the University of Colorado, Boulder, in 1990.

$\mathrm{He}$ is a Professor at Tsinghua University. His research interests are mainly in automatic control, intelligent robot, and computer vision.

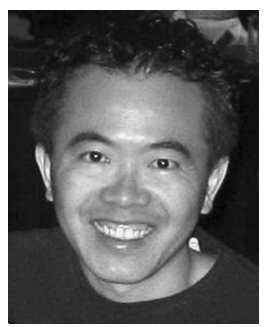

Ming-Hsuan Yang received the Ph.D. degree in computer science from the University of Illinois at Urbana-Champaign, Urbana, in 2000.

$\mathrm{He}$ is an Assistant Professor in the Department of Electrical Engineering and Computer Science, University of California at Merced. He was a Senior Research Scientist at Honda Research Institute working on vision problems related to humanoid robots. He coauthored the book Face Detection and Gesture Recognition for Human-Computer Interaction (Kluwer Academic, 2001) and edited the Special Issue on Face Recognition for Computer Vision and Image Understanding in 2003. He served as an Area Chair for the IEEE Conference on Computer Vision and Pattern Recognition in 2008 and 2009 and as a Publication Chair in 2010, as well as an Area Chair for the Asian Conference on Computer in 2009 and 2010. He served as an Associate Editor of the IEEE TRANSACTIONS ON PATTERn ANALYSIS AND MAChine INTElligence, and he is currently an Associate Editor of Image and Vision Computing.

Dr. Yang is a Senior Member of the Association for Computing Machinery. He was a recipient of the Google Faculty Award, the UC Merced Distinguished Early Career Research Award, and the National Science Foundation CAREER Award. 